\title{
Design and Implementation of Information Technology based Collaborative Learning Platform for College English Advising
}

\author{
CHEN Xian ${ }^{1, a}$ \\ ${ }^{1}$ Gannan Normal University, Ganzhou 341000,China \\ a chenxian_gnu@163.com
}

\begin{abstract}
Keywords: Information Technology (IT); College English; Collaborative Learning; System Optimization and Enhancement; Search Engine Optimization
\end{abstract}

\begin{abstract}
With the fast and bursting development in computer and web technology, it is essential for use to combine the state-of-the-art technique with the traditional English advising. Electronic learning, better known as e-learning, refers to the issues related to virtual distance education through electronic communication mechanisms, specifically the internet. It is based on the use of approaches with several formats and functionalities that may support the teaching-learning process, such as e-mail, web pages, forums, various learning plat-forms, and so on. The main advantages defined by studying through online tools include flexibility, convenience ease of access, consistency and repeatability of the proposed tasks. In this paper, we conduct research on designing and implementation of information technology based collaborative learning platform for college English advising. The proposed platform makes the breakthrough of developing a novel pattern for advising.
\end{abstract}

\section{Introduction}

Microteaching is a classroom teaching skills training methods for students in school and in-service teachers, which is defined as a Controllable practice system makes normal students and teachers could focus on solving a specific behavior or study under a controlled conditions, built on educational theory, visual theory and technology. With the development of modern open and distance education and its social influence of the constantly expanding, as one of the standards of modern distance education to construct learning resources construction has aroused the concern of the remote education workers. How to design and production of multimedia learning resources and to send, make course learning resources effectively to provide personalized support services for distance learners, thus reduce the dropout rate of distance learners, increase in the retention of scholars, also caused the thought of remote education workers. We define the concept of learning resources from the view of media development of education technologies [1-3]. The American association of education communication and technology (AECT) when in education technology to define multiple identified the meaning of learning resources: such as the 1977 think learning resources should not be limited to the definition of media, it should also include personnel, materials and equipment [4-5]; In 1999, the definition of learning resources to support system, teaching materials and the environment, namely all contribute to individual factors of effective learning. Self-rating professor think learning resources have points, as well as general learning resources in its narrow sense refers to the learning content and learning materials; and generalized learning resources is the floorboard of the learning support services, it includes not only the learning content and learning materials, and also includes the people, the media, elements such as strategy, method, environment and conditions. These resources include specifically designed for the realization of specific learning goals of resources; Also include in reality can be directly used to resources [6-7]. The combination of internet-based teaching, learning models and ESP courses provides a distinct interaction platform for learners. A one-year study on sixty students proves the fact that internet-based ESP teaching and learning models manage to play a positive role in prompting learner autonomy [8].

The advantages of this new computational paradigm with respect to other competing technologies are clear. First, cloud application providers strive to provide the same or better services and 
performance as if the software programs were installed locally on end-user computers, so the users do not need to spend money buying complete hardware equipment for the software to be used. In this paper, we conduct research on designing and implementation of information technology based collaborative learning platform for college English advising. In this way, three different levels are often distinguished, as we can observe in Figure 1. In the remainder of this section, we will first describe each of these three levels, providing the features that define each of them, and some examples of the most well-known systems of each type [9].

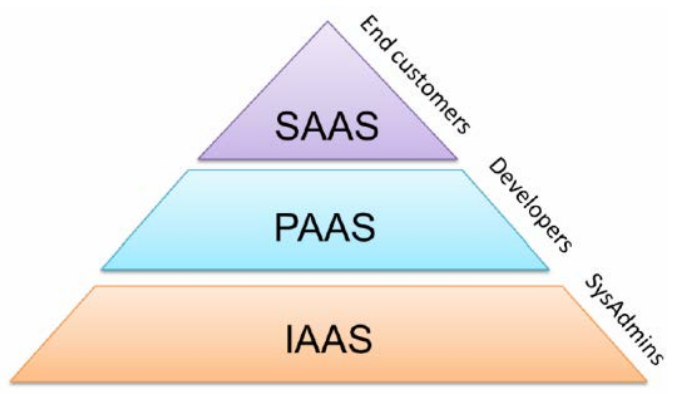

Fig.1 The illustration of our system layers

\section{Our Designed System and Function Description}

Pre-Discussion for the Platform. As we stated in the introduction of this work, with the huge growth in the number of students, educational content, services that can be offered and re-sources made available, e-learning system dimensions grow at an exponential rate. The challenges with regard to optimizing resource computation, storage and communication requirements, and dealing with dynamic concurrency requests highlight the necessity of the use of a platform that meets scalable demands and cost control. This environment is cloud computing. This guideline and Bruner advocated by the "discovery method" teaching idea is consistent, namely course outside its inherent structure, should provide enough space for the students, the opportunity and platform to actively discover and learn new knowledge. Cannot blindly infusion teaching information, but guide the learners to their experiences into the learning process, learners should be the construction of "meaning" and "solutions". At the same time, the characteristics of the Internet with its timeliness, richness and diversity are widely applied to English teaching. Among them, around how to use the Internet to cultivate student ability of autonomic learning research has become the hotspot of the reform of English teaching. The basic architecture is shown in the figure 2.

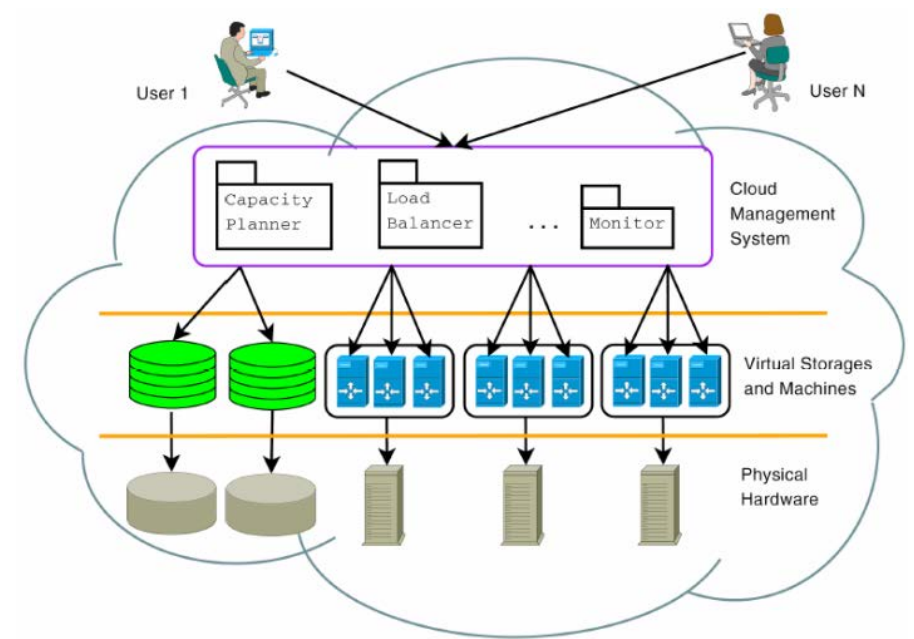

Fig.2 The illustration of Information Technology based English Advising Platform

The Detailed Structure of the Platform. Under these circumstances, a private cloud is mostly preferred for three reasons: First, educational centers have the option of utilizing their existing infrastructure which allows them to have a better control over the resources. Regarding this previous 
point, this implies a low cost process for setting up the cloud. Finally, the security issue is a major factor in the preference for this type of cloud. Certain activities such as the admission process for new students and the policies thereof, examinations/tests conducted for existing students and all research activities re-quire confidentiality and breaches in security cannot be tolerated. In formal learning resources and media selection, the British scholar riding lunatic think education technology, the present forms of teaching resources will impact on learners' learning. Because the learner's cognitive style and cognitive structure is different, the teaching resources are different forms of preference. Especially for the low learning ability and information processing ability is limited, the learners, learning resources present form, the matching of cognitive structure and cognitive style is more critical. This definition in essence emphasizes the students as the main body in the learning process, based on the three-dimensional network platform, can according to its own characteristics, such as interest, hobby, one's current level of language, the demand for knowledge of the target language, set learning goals, choose from a variety of network resources learning contents, select learning methods and strategies. In the process, students' main body consciousness can be fully stimulated, learning autonomy can also be effectively. The combination of cloud technologies and e-learning has been insufficiently explored. The pedagogical possibilities of the collaborative aspect of the cloud are studied in Stevenson and Hedberg. In the aforementioned work, the authors refer to the lack of research that might provide a theoretical framework with which pedagogy could be developed. However, the mobility inherent in the cloud approach could be stressed as a major advantage in developing conceptual frameworks and establishing effective teaching and learning strategies. Regarding the use of cloud computing for educational purposes, we can distinguish between the public cloud and the private cloud solutions. It is a framework which provides on-demand creation and configuration of VM images so that students are able to have their own Java servlet environment for experimentation, containing MySQL, Tomcat, PHP, and Apache web server. The architecture of the proposed system is shown in the figure 3.

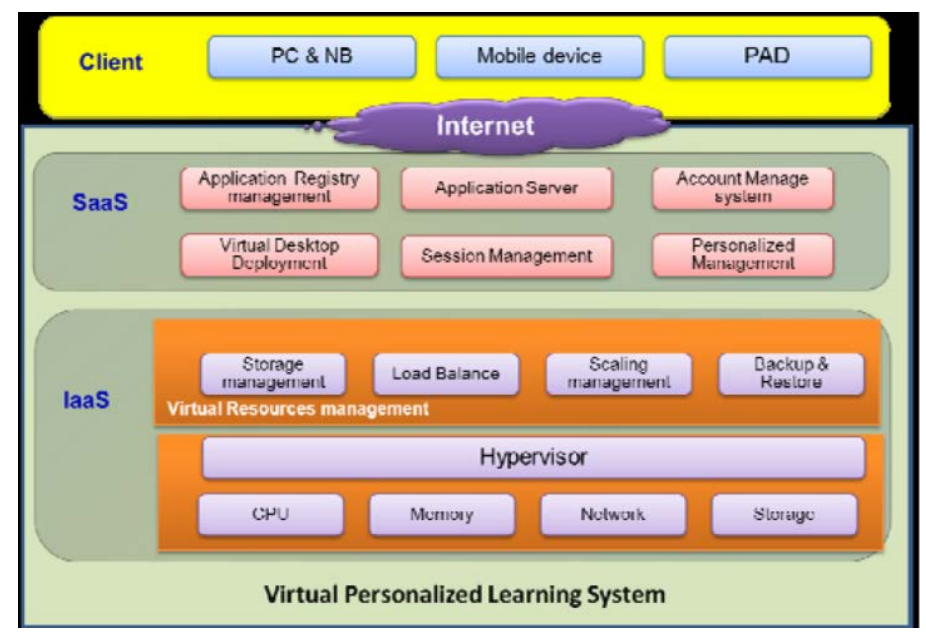

Fig.3The Architecture of Our Platform

Optimization of Current Platform with Data Analysis. The EDM process follows the same steps as general data mining (see Figure 4). First, it starts with the preprocessing of the data in order to prepare it for the learning stage, i.e., using cleaning techniques, reducing the number of instances and/or input variables to facilitate the working of the algorithms, and so on. Then, the learning algorithms are applied to extract useful information from the data, whose type will depend on the final aim of the user, such as classification, clustering, association rule mining, sequential mining or text mining. Finally, a post-processing step can be carried out to enhance the obtained results or to provide a more interpretable representation of the system. . In 2006, the simulation microteaching is completely upgrading to digital micro, oriented to all teaching professional. This digital system consists of a main control room and 16 micro classrooms, using C / S architecture. Compared with previous simulation, the digital micro teaching device are easy to use, video playback flexible and resource easy to replication, so well received by teachers and students. Innovative teaching methods 
are the key to improving teaching quality. When the teaching aim and contents is confirmed, whether the teaching methods are appropriated is very important for student to understand knowledge, develop abilities and train skills. Because micro-teaching is an innovative teaching method itself which determining that it must have a huge difference from all kinds of traditional teaching forms, it can push the reform of lesson system and content. The great advantage of the EDM methodology is its use in research and in building models in various areas that can influence e-learning systems. To optimize the system we could use mathematical tools introduced in the literatures [8-10].

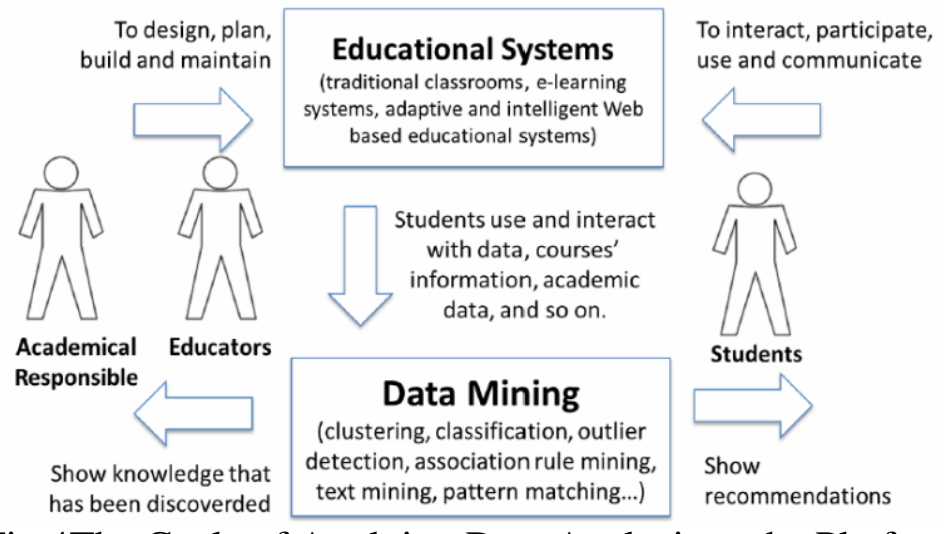

Fig.4The Cycle of Applying Data Analysis to the Platform

\section{Conclusion and Summary}

Electronic learning, better known as e-learning, refers to the issues related to virtual distance education through electronic communication mechanisms, specifically the internet. It is based on the use of approaches with several formats and functionalities that may support the teaching-learning process, such as e-mail, web pages, forums, various learning plat-forms, and so on. The main advantages defined by studying through online tools include flexibility, convenience ease of access, consistency and repeatability of the proposed tasks. In addition, the demand for teaching resources is usually dynamic and rapid way each are not identical, to activity heights. To overcome the difficulties that the process of English broadcasting for college students. In this research paper, we conduct research on designing and implementation of information technology based collaborative learning platform for college English advising. As the result, we help to modify the general process of college collaborative learning effect.

\section{References}

[1] Bieliková, Mária, Marián Šimko, Michal Barla, Jozef Tvarožek, Martin Labaj, Róbert Móro, Ivan Srba, and Jakub Ševcech. "ALEF: from application to platform for adaptive collaborative learning." In Recommender Systems for Technology Enhanced Learning, pp. 195-225. Springer New York, 2014.

[2] Popescu, Elvira. "Providing collaborative learning support with social media in an integrated environment." World Wide Web 17.2 (2014): 199-212.

[3] Cen, Ling, et al. "Learning alone or in a group-An empirical case study of the collaborative learning patterns and their impact on student grades." Interactive Collaborative Learning (ICL), 2014 International Conference on. IEEE, 2014.

[4] Serrano-Cámara, Luis Miguel, et al. "An evaluation of students' motivation in computer-supported collaborative learning of programming concepts." Computers in Human Behavior 31 (2014): 499-508. 
[5] Göhnert, Tilman, et al. "Integration of a Flexible Analytics Workbench with a Learning Platform for Medical Specialty Training." LAK Workshops. 2014.

[6] Violante, Maria Grazia, and Enrico Vezzetti. "Implementing a new approach for the design of an e - learning platform in engineering education." Computer Applications in Engineering Education 22.4 (2014): 708-727.

[7] Wan, H. T., and D. J. Tsai. "Building a community-based cooperative e-learning platform for Taiwanese tribal elders." Gerontechnology 13.2 (2014): 295.

[8] Challco, Geiser Chalco, et al. "Towards an Ontology for Gamifying Collaborative Learning Scenarios." Intelligent Tutoring Systems. Springer International Publishing, 2014.

[9] Xu, Biao, Xu-Huan Wang, Wei Wei, and Haoxiang Wang. "On reverse Hilbert-type inequalities." Journal of Inequalities and Applications 2014, no. 1 (2014): 1-11.

[10]Johnson-Glenberg, Mina C., et al. "Collaborative embodied learning in mixed reality motion-capture environments: Two science studies." Journal of Educational Psychology 106.1 (2014): 86. 